\title{
Rursus
}

Russus

Poiétique, réception et réécriture des textes antiques

2| 2007

Le modèle animal (II)

\section{Les représentations animales chez Horace dans le livre II des Satires et les livres I à III des Odes : présence ou absence d'une transformation générique}

Dominique Voisin

\section{(2) OpenEdition}

\section{Journals}

Édition électronique

URL : http://journals.openedition.org/rursus/100

DOI : 10.4000/rursus. 100

ISSN : 1951-669X

Éditeur

Université Nice-Sophia Antipolis

Référence électronique

Dominique Voisin, « Les représentations animales chez Horace dans le livre II des Satires et les livres I à III des Odes : présence ou absence d'une transformation générique », Rursus [En ligne], 2 | 2007, mis en ligne le 04 mai 2007, consulté le 15 novembre 2019. URL : http://journals.openedition.org/rursus/ $100 ;$ DOI : 10.4000/rursus.100

Ce document a été généré automatiquement le 15 novembre 2019.

Rursus 


\title{
Les représentations animales chez Horace dans le livre II des Satires et les livres I à III des Odes : présence ou absence d'une transformation générique
}

\author{
Dominique Voisin
}

1 Le choix d'un tel sujet répond aux exigences à court terme du Lalia, le thème de l'animalité, mais également à son projet à long terme, l'étude de la réécriture. Par ailleurs, les études récentes sur les représentations animales chez Horace sont quasiment inexistantes ${ }^{1}$ et ne concernent par la question de la transformation, selon le genre choisi, de ces représentations.

2 Quant au choix des textes, s'il obéit à la une nécessité technique de limiter le corpus étant donné les contraintes imposées par les conditions matérielles de cette recherche, il répond également, et plus justement, à l'espoir de réduire, dans la mesure du possible, les différences dues à l'évolution dans le temps des représentations animales chez Horace : le deuxième livre des Satires, publié en 29, a été composé entre 33 et 30; les trois premiers livres des Odes, publiés en 23 ont été composés entre 33 et 23 . Ceci devrait permettre de mieux apprécier les différences, si elles existent, relatives au genre employé. C'est pour cette dernière raison que j'ai exclu les Epodes de la comparaison: par nature, elles comportent en effet des ressemblances sensibles de contenu et de forme tant avec les Satires qu'avec les Odes. En revanche, une étude comparative similaire pourrait être menée à partir des Odes et des Epîtres.

3 J'ai préféré partir des faits, et de faits facilement identifiables : j'ai donc fondé cette étude sur un catalogue des représentations animales, sans pour autant me satisfaire d'une description ou d'une classification, mais je me suis interrogée sur leurs formes et leurs raisons d'être. Il m'a semblé cependant qu'il valait la peine d'alourdir cet article par la présentation de ce catalogue. La façon dont il a été établi, loin d'être innocente, 
oriente la suite de l'investigation : autant dès lors le soumettre au jugement critique des lecteurs afin de leur permettre d'apprécier les fondements mêmes de l'étude proposée. D'autre part, un tel recueil peut être utile, dans la mesure où il permet de savoir rapidement que telle représentation animale figure chez tel auteur, sous telle forme et avec telle fréquence.

Pour établir ce catalogue, j'ai d'abord identifié les représentations au sein même des textes, y compris quand celles-ci n'étaient pas explicitement données comme des représentations de l'animal pour lui-même, telles les images, comparaisons et métaphores. Dans ce dernier cas, la difficulté venait des métaphores usées. Toute langue comprend nombre d'images banalisées, sinon lexicalisées, qui ne renvoient plus à un animal donné (telles les "ailes de la Fortune »). J'ai donc exclu les métaphores dont le TLL signalait la fréquence et par conséquent la banalité chaque fois que leur usage, en situation, l'autorisait. En revanche toute métaphore morte revivifiée par le contexte a été traitée.

5 Restaient à classer les représentations animales une fois qu'elles avaient été isolées. Dans le souci d'éviter l'anachronisme et de prêter à Horace des modes de pensée étrangers à l'esprit antique, j'ai choisi de suivre le classement d'un Romain du siècle suivant, Pline l'Ancien, qui reste l'auteur de l'exposé le plus circonstancié en langue latine sur le monde animal. Dans son Histoire naturelle, où il entend mettre à la disposition «du grand public, la foule des agriculteurs, celle des artisans, ceux que n'absorbent pas des études » les connaissances relatives à la natura, il consacre quatre livres aux animaux (VIII-XI). Il semble raisonnable de penser que cette encyclopédie de vulgarisation n'était pas fort éloignée des savoirs et des modes de pensée des auditeurs cultivés contemporains d'Horace. L'absence ou la présence de ces mêmes animaux chez Horace peut être une première indication de la manière originale dont le poète traite les représentations animales².

\section{1-Le catalogue des représentations animales dans le livre 2 des Satires et les livres 1 à 3 des Odes classé selon le catalogue plinien de l'Histoire naturelle ${ }^{3}$}

\section{Les animaux terrestres ${ }^{4}$}

Bêtes fauves ou sauvages en général

\begin{tabular}{|l|l|}
\hline Satires & Odes \\
\hline \hline II, 7, v. 70-71 & $\begin{array}{l}\text { I, } 12, \text { v. } 22-23 \\
\text { III, } 3, \text { v. } 40-42\end{array}$ \\
\hline
\end{tabular}

Serpents (14)

\begin{tabular}{|l|l|}
\hline Satires & Odes \\
\hline
\end{tabular}




\begin{tabular}{|c|c|}
\hline II, 8 , v. 94-95 & $\begin{array}{l}\text { I, } 8: \text { v. } 8-10 \\
\text { I, } 17 \text {, v. } 8 \\
\text { I, } 37, \text { v. } 26-28 \\
\text { II, } 13 \text {, v. } 35-36 \\
\text { II, } 19 \text {, v. } 19-20 \\
\text { III, } 4 \text {, v. } 17-18 \\
\text { III, } 10 \text {, v. } 18 \\
\text { III, } 11 \text {, v. } 15-20 \\
\text { III, } 27 \text {, v. } 5\end{array}$ \\
\hline
\end{tabular}

$7 \quad$ [Bisons, ures (15) - Elan, achlis, bonasus (16)]

LIONS (17-22)

\begin{tabular}{|c|c|}
\hline Satires & Odes \\
\hline II, 3, v. 186 & $\begin{array}{l}\text { I, } 16 \text {, v. } 13-16 \\
\text { I, } 22, \text { v. } 15-16 \\
\text { I, } 23 \text {, v. } 10 \\
\text { II, } 13 \text {, v. } 40-41 \\
\text { II, } 19 \text {, v. } 23-24 \\
\text { III, } 2 \text {, v. } 10-12 \\
\text { III, } 11 \text {, v. } 41-42 \\
\text { III, } 20 \text {, v. } 1-2 ; 9-10: \\
\text { III, } 27 \text {, v. } 51-52 \\
\text { III, } 29 \text {, v. } 19\end{array}$ \\
\hline
\end{tabular}

Panthères (23-24)- TIgRES (25)

\begin{tabular}{|l|}
\hline Odes \\
\hline I, 23 , v. 9 \\
III, 3, v. 13-15 \\
III, 11 , v. 13-14 \\
III, 27 , v. 55-56 \\
\hline
\end{tabular}

8 [Chameaux (26)-Girafes (27) - Loup-cervier, cèphes (28) - Rhinocéros (29)]

Lynx(30): [sphinx, crocottes, cercopithèques (30 - 32)]

Odes 
II, 13 , v. 40-41

[Basilics (33)]

Loups (34)

\begin{tabular}{|l|l|}
\hline Satires & Odes \\
\hline II, 1, v. 52 ; 54-55 & I, 15, v. $29-31$ \\
II, 2 , v. 64 & I, 17, v. 9 \\
& $\begin{array}{l}\text { I, } 22, \text { v. } 9-16 \\
\text { III, } 18, \text { v. 5, 9-13 } \\
\text { III, 27, v. 2-3 }\end{array}$ \\
\hline
\end{tabular}

10 [Mangouste (36) - Crocodile (37) - Scinque ou varan (38) - Hippopotame (39-40)Hyènes (44)- Corocottes (45)- Mantichores (45)- Onagres - (46) - Castors, loutres, veaux marins, stellions (47-49)]

Cerfs (50)

\begin{tabular}{|l|}
\hline Odes \\
\hline I, 1 , v. $25-28$ \\
I, 15 , v. $29-31$ \\
I, 23 , v. $1-3 ; 6-7 ; 9-10$ \\
II, 16, v. 23 \\
III, 5, v. $31-32$ \\
III, 12 , v. $10-12$
\end{tabular}

11 [Caméléons (51) - Renne, lycaon, thos (52) - Porc-épic (53)]

OURS (54)

Odes

III, 4, v. 17-18

RATS (55)

\begin{tabular}{|l} 
Satires \\
\hline II, 3, v. 247 \\
II, 6, v. 79-117
\end{tabular}


12 [Hérissons (56) - Léontophonon, lynx (57) - Blaireaux, écureuils (58)]

Escargots (59)

Satires

II, 4, v. 59-60

LÉZARDS $(60)$

Odes

I, 23 , v. 6-7

CHIENS (61-63)

\begin{tabular}{|l|l|}
\hline Satires & Odes \\
\hline II, 1, v. 84-85 & I, 1, v. 25-28 \\
II, 2, v. 64 & II, 13 , v. $34-35$ \\
II, 3 , v. 259 & II, 19, v. 29-32 \\
II, 5 , v. 83 & III, 11 , v. 15-20 \\
II, 6, v. 114-115. & III, 16, v. 1-2 \\
& III, 27, v. 1-2 \\
\hline
\end{tabular}

CHeVAuX (64-67)

Satires Odes 


\begin{tabular}{|c|c|}
\hline $\begin{array}{l}\text { II, } 1 \text {, v. } 15 ; 19-20 ; 26 \\
\text { II, } 2 \text {, v. } 9-10\end{array}$ & $\begin{array}{l}\text { I, } 6, \text { v. } 3-4 \\
\text { I, } 8, \text { v. } 5-7 \\
\text { I, } 12 \text {, v. } 25-27 \\
\text { I, } 15 \text {, v. } 24-25 \\
\text { I, } 15, \text { v. } 9-10 \\
\text { I, } 25 \text {, v. } 13-14 \\
\text { II, } 1 \text {, v. } 19-20 \\
\text { II, } 16 \text {, v. } 34-35 \\
\text { III, } 1 \text {, v. } 40 \\
\text { III, 3, v. } 15-16 \\
\text { III, } 4 \text {, v. } 34 \\
\text { III, 7, v. } 25-26 \\
\text { III, } 11 \text {, v. } 7-10 \\
\text { III, } 24 \text {, v. } 54-56 \\
\text { III, } 27 \text {, v. } 7\end{array}$ \\
\hline
\end{tabular}

[Anes - Mules] (68-69)

Bœufs, vaches, taureaux (70-71)

\begin{tabular}{|c|c|}
\hline Satires & Odes \\
\hline $\begin{array}{l}\text { II, 1, v. } 52 ; 54-55 \\
\text { II, 3, v. 199-200 ; 314-320 } \\
\text { II, 7, v. 91-94 }\end{array}$ & $\begin{array}{l}\text { I, } 10, \text { v. } 9-10 \\
\text { I, } 31, \text { v. } 5-6 \\
\text { I, } 36, \text { v. } 1-3 \\
\text { II, } 5 \text {, v. } 1-9 ; 15-19 \\
\text { II, } 8 \text {, v. } 21 \\
\text { II, } 14 \text {, v. } 5-7 \\
\text { II, } 16, \text { v. } 33-34 \\
\text { III, 3, v. } 40-42 \\
\text { III, } 6 \text {, v. } 42-43 \\
\text { III, } 11, \text { v. } 41-42 \\
\text { III, } 13 \text {, v. } 10-12 \\
\text { III, } 18, \text { v. } 5 ; 9-13 \\
\text { III, } 21 \text {, sv. } 18 \\
\text { III, } 27 \text {, v. } 25-26 ; 45-48 ; 71-72\end{array}$ \\
\hline
\end{tabular}

Petit bétail : ovins, moutons, agneaux, béliers, brebis, moufflon (72-75)

\begin{tabular}{|l|l|}
\hline Satires & Odes \\
\hline
\end{tabular}




\begin{tabular}{|l|l|}
\hline II, 2 , v. $114-115$ & I, 4, v. $11-12$ \\
II, 3, v. $197 ; 202 ; 211 ; 214 ; 219$ & I, 4, v. 3 \\
II, 5, v. 7 & II, 17, v. $30-32$ \\
II, 6, v. 14-15 & II, 6, v. 10-11 \\
& III, 13, v. $10-12$ \\
& III, 16, v. 33 \\
& III, 18, v. $5,9-13$ \\
& III, 29, v. $21-22 ; 37$ \\
\hline
\end{tabular}

Chèvres, boucs (76)

\begin{tabular}{|l|l|}
\hline Satires & Odes \\
\hline \hline II, 2, v. 121-122 & $\begin{array}{l}\text { I, } 17, \text { v. } 2-9 \\
\text { III, 8, v. 6-7 } \\
\text { III, } 13 \text {, v. } 3-8 \\
\text { III, } 15, \text { v. } 12 \\
\text { III, } 18, \text { v. } 5 \text {; 9-13 }\end{array}$ \\
\hline
\end{tabular}

PORCs (77)

\begin{tabular}{|l|l|}
\hline Satires & Odes \\
\hline \hline II, 3, v. 164-165 & III, 22, v. 7-8 \\
& III, 23, v. 3-4 \\
\hline
\end{tabular}

Sangliers (78-79)

\begin{tabular}{|l|l|}
\hline Satires & Odes \\
\hline \hline II, 2, 41-43; 89 & I, 1, v. 25-28 . \\
II, 3, v. 73 ; 245 & III, 12 , v. 10-12 \\
II, 4, v. 40-42 & \\
II, 8, v. 6-7 & \\
\hline
\end{tabular}

Daims (79)

\begin{tabular}{|l|}
\hline Odes \\
\hline I, 2 , v. 7-12 \\
\hline
\end{tabular}


14 [Singes] (80)

Lièvres (81)

\begin{tabular}{|l|l|}
\hline Satires & Odes \\
\hline II, 2, v. 9-10 & I, 37, v. 17-20 \\
II, 4, v. 44 & \\
II, 8, v. 89-90 & \\
\hline
\end{tabular}

Grenouilles (83)

Satires

II, 3, v. 314-320

Chevreuil (83)

Satires

II, 4, v. 43

Renards (83)

\begin{tabular}{|l|l|}
\hline Satires & Odes \\
\hline II, 3, v. 186 & III, 27, v. 4 \\
\hline
\end{tabular}

\section{Les animaux aquatiques ${ }^{5}$}

\section{Poissons en général}

\begin{tabular}{|l|l|}
\hline Satires & Odes \\
\hline II, 2 , v. 16-17 & I, 2 , v. 7-12 \\
II, 3, v. 245 & III, 1, v. 33-34 \\
II, 4, v. 37 ; 45-46; 76-77 & \\
II, 5 , v. 24-25 & \\
II, 8, v. 27-30; 46 & \\
\hline
\end{tabular}


15 [Tritons, Néréides, éléphants de mer (4) - Baleines, orques (5) - Dauphins (7) Thyrsions (11) - Tortues (12-13) - Veaux marins ou phoques (14)]

Cordyles, pélamydes, THONS, apolectes, cybies (18)

Satires

II, 5 , v. 44

16 [Amies, maquereaux- Espadons (21)]

Mulet (26)

Satires

II, 2 , v. $33-34 ; 37 ; 39$

Esturgeon (27)

Satires

II, 2, v. 47-49

LOUP ou bar, aselle (28)

Satires

II, 2, v. 31-36

SCARE, lotte (29)

Satires

II, 2, v. 21-22

17 [Rougets, sargues (30)]

Turbots et plies (36)

Satires

II, 2, v. 41-43 ; v. 47-49 ; v. 95-96

II, 8 , v. $27-30$ 
18 [Anguilles (38)]

Murènes (39)

Satires

II, 8, v. $42-43$

19 [Rémora (41) - Hirondelle de mer, poisson cornu, dragon de mer (43) - Seiche, calmar, peignes (45) - Poulpe (46-48) - Argonaute (49) - Crustacés (50-52) - Langouste (50)]

Crabes, pinnotères, ouRsINs, escargots, PEIgNES (51)

Satires

II, 4, v. 33-34:

II, 8, v. 52-53

Coquillages en général

Satires

II, 2, v. 73-74

II, 4 , v. $28,30-31$

II, 8 , v. $27-30$

20 [Perles (54-59]

MUREX (60-65)

\begin{tabular}{|l|l|}
\hline Satires & Odes \\
\hline II, 4, v. 32 & II, 16, v. 35-37 \\
\hline
\end{tabular}

21 [Pinnes et pinnotères (66) - Torpille, pastenague, scolopendres, silure, poisson bélier (67) - Orties de mer (68) - Eponges (69) - Chiens de mer (70)]

Squilles (74)

Satires

II, 4, v. 58-59

II, 8 , v. $42-43$ 
HUîTRES $(79-80)$

Satires

II, 2, v. 21-22

II, 4 , v. 33

[Anthias (85) - Etoiles de mer (86) - Couteaux (87)]

\section{Les oiseaux ${ }^{6}$}

Oiseaux en général

\begin{tabular}{|l|l|}
\hline Satires & Odes \\
\hline II, 3, v. 45-46; 73 & I, 34, v. 14-16 \\
II, 8, v. 27-30 & $\begin{array}{l}\text { III, 1, v. 20-21 } \\
\text { III, 2, v. 21-24 } \\
\text { III, 29, v. 53-54 }\end{array}$ \\
\hline
\end{tabular}

23

[Autruches (1) - Phénix (2) - Aigles (3-6) - Vautour (7) - Sanquale, immusule (8) Eperviers, Buses - Milans (9-12)]

CORNEILLES, orfraie (14)

\begin{tabular}{|l|}
\hline Odes \\
\hline III, 17 , v. $12-13$ \\
III, 27 , v. $1-2 ; 9-10 ; 15-16$
\end{tabular}

Corbeaux (15)

\begin{tabular}{|l|l|}
\hline Satires & Odes \\
\hline II, 5, v. 55 & III, 27, v. 11-12 \\
\hline
\end{tabular}

24 [Grands-ducs - Chouettes (19)]

Piverts (20)

Odes

III, 27 , v. 15-16 
PAONS (22-23)

Satires

II, 2, v. 31-34

25 [Coqs (24-25)]

OIES $(26-27)$

Satires

II, 8 , v. 88

[Commagène (28) - Chénalopèces, souchets, coqs de bruyère, outardes (29)] - GRUES (30)

Satires

II, 8, v. 86-87

Cigognes (31)

Satires

II, 2, v. 47-49

CYgNes (32)

Odes
I, 6 , v. 2
II, 20 , v. $1-2 ; 9-12 ; 15-16$
III, 28 , v. $14-15$

[Cailles, glottis, cychrames, moyens ducs (33)]

Hirondelles, gRIVES, MERLES, étourneaux (34)

$$
\begin{array}{|l|}
\text { Satires } \\
\hline \text { II, 2, v. 73-74 } \\
\text { II, 8, v. } 91
\end{array}
$$


Tourterelles, ramiers , éperviers (35)

\begin{tabular}{|l|}
\hline Odes \\
\hline I, 2, v. 7-12 \\
I, 37, v. 17-20
\end{tabular}

27 [Loriots, huppes (36) - Memnonides (37) - Méléagrides (38) - Séleucides (39) - Ibis (40)]

Rossignols (43)

Satires

II, 3, v. 245

28 [Becs-figues, rouges-gorges, queues-rouges (44) - Oenanthes, loriots (45)]

Alcyons, PLONgEONS (47)

Satires

II, 2, v. 47-49

29 [Hirondelles (49) - Acanthyllis (50) - Guêpier, perdrix (51)]

Pigeons / colombes (52-53)

\begin{tabular}{|l|l|}
\hline Satires & Odes \\
\hline II, 2, v. 21-22 & III, 4, v. 9-13 \\
II, 8, v. 91 & \\
\hline
\end{tabular}

30 [Apodes ou martinets (55) - Tette-chèvres, spatules (56) - Chardonnerets, butors, anthus (57) - Perroquets (58) - Pies glandaires (59) - Oiseaux de Diomède (61) - Porphyrion (63) - Himantopodes (64) - Pélicans (66) - Phalérides, faisans, poules numidiques (67)]

Flamants, francolins, phalacrocorax, LAgOPÈDES (68)

Satires

II, 2, v. 21-22

$31 \quad$ [Vivions (69)] 
POULES (71)

Satires

II, 2, v. $32 ; 121-122$

II, 4 , v. 18-19

EuFs (74-77)

32 [Hérons (79)]

33 [Serpents (82) - Salamandres (86)]

Les insectes

Abeilles (4-23)

Odes

III, 16 , v. 33

[Frelons et guêpes (24) - Bombyx d'Assyrie (25) - Bombyles, necydalles (26) - Bombyx de Cos (27) - Araignées (28-29) - Scorpions (30) - Stellions (31) - Cigales (32) - Scarabées, lampyrides (34) - Sauterelles (35) - Fourmis (36) - Chrysalides, asilus, papillons (37)]

TEIgNES, cantharides, cousins (41)

Satires

II, 3 , v. 119-120

[Pyrallis ou pyrotès (42) - Ephémères (43)]

\section{2-Un premier bilan, quantitatif, semble nécessaire ; quel est le nombre d'animaux en valeur absolue et en valeur relative respectivement dans le livre II des Satires et dans les livres I à 3 des Odes?}

\section{Voyons d'abord le nombre d'animaux en valeur absolue}

Cinquante animaux différents sont cités dans les Satires contre trente-trois dans les Odes (pour simplifier je parlerai dorénavant seulement de Satires et d'Odes pour désigner le livre II des Satires et les livres I à 3 des Odes). Cela représente en moyenne 6,25 animaux par satire contre 0,37 par ode, soit à peine $6 \%$ de l'effectif de chaque satire. Mais il faut corriger cette disproportion globale en envisageant trois facteurs : 

chaque genre. Les 8 satires du livre II totalisent 1083 vers et varient de 86 vers pour la plus courte à 326 vers pour la plus longue; les 88 odes totalisent 2453 vers seulement (de 8 vers pour la plus courte à 80 pour la plus longue). Si l'on compare le nombre d'animaux différents par rapport au nombre de vers, on obtient un total de 4,61 \% pour les satires, et de $1,34 \%$ pour les odes, soit près de $29 \%$ de l'effectif des satires ; si l'on corrige ce résultat en ne tenant compte que des odes concernées, on arrive à un total de $2 \%$ pour les odes, soit près de $44 \%$ de l'effectif des satires. En appliquant le même calcul au nombre d'apparitions animales, on obtient un rapport de $8,58 \%$ pour les satires, de $4,81 \%$ pour les odes en général et de $7,17 \%$ pour les odes concernées, soit quasiment $84 \%$ de l'effectif des satires.

Tout d'abord, toutes les satires mentionnent des animaux alors que seuls $58 \%$ des odes sont concernés. Si l'on ne tient compte que de ces dernières, la moyenne est de 0,64 animal par ode, soit un peu plus de $10 \%$ de l'effectif de chaque satire.

d'animal, même quand il s'agit de la même bête, il n'y a plus que 92 apparitions animales dans les satires, soit 11, 5 par satire contre 113 apparitions dans les odes, soit 1, 28 par ode en général ou 2,21 par ode concernée c'est-à-dire près de $20 \%$ de l'effectif des satires.

Ainsi, du point de vue de la présence animale dans les œuvres, la différence quantitative entre les satires et les odes est moins grande qu'il n'y paraît; on peut déjà conclure cependant à une plus grande unité formelle du genre lyrique dans l'exploitation de l'espèce animale par rapport à la "macédoine bigarrée » des cinquante animaux différents du genre satirique.

\section{Passons maintenant à l'analyse quantitative appliquée à chaque genre}

Bien que les Satires mentionnent toutes un animal, elles offrent une grande diversité dans le traitement quantitatif de l'espèce en question. Les plus pauvres, au nombre de 4 , offrent seulement 2 à 6 apparitions animales et présentent un total de 2 à $7 \%$ par rapport au nombre de vers; les plus riches offrent entre 16 et 25 apparitions animales et présentent un total, par rapport au nombre de vers, qui varie entre 17 et $19 \%$, soit près de 10 fois plus que le pourcentage le plus faible: il s'agit de 3 satires qui ont en commun un long développement quasi exclusif concernant la nourriture : le pseudotraité de l'art du goût de Catius, dans la satire 4, l'éloge de la vie rurale du campagnard Ofellus dans la satire 2 et le souper ridicule de Nasidiénus dans la satire 8. Le cas de la satire 3 est un peu particulier: dans ses 326 vers, elle offre jusqu'à 18 apparitions animales ; mais celles-ci ne représentent que 5,5\% par rapport à la masse des vers ; c'est que la verbeuse critique de la folie contemporaine développée par l'ex-collectionneur et stoïcien nouvellement converti Damasippe use quelquefois de proverbes familiers et qu'y figure, bien évidemment, une brève dénonciation du luxe de la table.

42 Les Odes, nous l'avons vu, pour 37 d'entre elles, ne mentionnent aucun animal. Quant aux odes concernées par notre sujet, la brièveté relative de chacune fait que le nombre d'animaux y coïncide avec celui des apparitions animales. Le nombre de ces apparitions varie, de manière générale, entre 1 et 4 ( 1 apparition dans 24 odes ; 2 apparitions dans 10 odes; 3 apparitions dans 8 odes; 4 apparitions dans 3 odes) et présente donc une 
relative stabilité; une ode (III, 27) offre jusqu'à 12 apparitions animales, ce qui représente un total de 16\% par rapport au nombre de vers (76) (rappel du total moyen des odes concernées : $4,81 \%$ ), tandis que le total de l'ode la moins bien pourvue en valeur absolue (1) et relative (III, 24) est de $1,56 \%$ soit près de 10 fois moins. Une ode est encore plus riche en valeur relative (I, 23) : avec ses 4 apparitions en 12 vers, elle offre un total de 33,33\% qui est près de 20 fois supérieur au total le plus faible.

Entre chaque livre des Odes, les représentations animales semblent inégalement réparties: en effet, le livre III compte 58 apparitions animales dans 24 odes sur 30 soit dans $80 \%$ des poèmes; le livre II n'en présente que 20 dans 10 odes sur 20 soit $50 \%$ des poèmes ; et le livre I compte 35 apparitions pour 17 odes sur 38 , soit moins de $45 \%$ des poèmes. Le troisième livre, dit des «odes civiques" serait-il plus d'une fois et demie plus « animalier » que les autres ? Là encore, il faut nuancer cette impression en tenant compte du nombre de vers et en calculant en fonction des seules odes concernées par les représentations animales; en effet, le lecteur est sensible autant, sinon plus, à la «densité animale » de chaque ode, plutôt qu'à celle de chaque livre. Ce sont alors les odes du livre I qui semblent les plus « animalières », avec un total de 7,6\%, puis celles du livre II avec un total de 6,5\%, enfin celles du livre III avec un total de 6,1\%

Certes, les Odes, dans un cas exceptionnel, offrent une disparité encore plus grande que les Satires dans la répartition des animaux entre chaque poème; pourtant, de manière générale, elles présentent une plus grande régularité, ce qui contribue, là encore, à l'unité formelle du genre lyrique.

\section{Regardons ensuite le bilan quantitatif relatif à chaque forme d'expression}

45 J'ai défini neuf formes d'expression qui permettent de traiter l'ensemble des représentations animales : soit l'animal est cité pour lui-même : $1^{\circ}$ - dans une simple mention, $2^{\circ}$ - dans une mention accompagné d'un élément descriptif, $3^{\circ}$ - dans une action ou une description d'au moins un vers dont il forme l'élément principal; soit l'animal sert de référence imagée ou allégorique : $4^{\circ}$ - dans une métaphore réduite, $5^{\circ}$ dans une comparaison réduite, $6^{\circ}$ - dans une métaphore filée, $7^{\circ}$ - dans une comparaison développée, $8^{\circ}$ - dans un court proverbe, $9^{\circ}$ - dans un apologue.

La simple mention domine dans les Satires (32 occurrences contre 19 dans les Odes), suivie de près par la mention descriptive ( 34 occurrences contre 43 dans les Odes). Les Satires ont l'exclusivité du proverbe (5 occurrences) et de l'apologue (2 occurrences).

Les Odes se plaisent à la vignette descriptive (28 actions ou descriptions longues contre 11 dans les Satires), usent de comparaisons et de métaphores réduites (respectivement 7 et 11 occurrences contre 1 et 2 occurrences dans les Satires), développent comparaisons et métaphores (respectivement 7 et 4 occurrences contre 4 et 1 dans les Satires). Certains animaux ont le privilège d'être traités sous différentes formes, jusqu'à 6 pour le cheval, 5 pour les chèvres et chevreaux, 4 pour les taureaux, les lions, les cerfs et les serpents : la forme la plus simple se charge alors de toutes les connotations de la forme la plus complexe : ainsi le cerf, dont les Odes III, 12 et I, 15, III, 5 ont décrit la fuite haletante et la faiblesse craintive, est employé de manière originale dans l'Ode II, 16 « le morbide souci n'abandonne pas, plus rapide que les cerfs " pour suggérer un double mouvement de fuite absurde: celui qui a fui la sagesse dans la poursuite des vains plaisirs de l'ambition ou de la richesse, absurdement, cherche à se dérober aux soucis 
de cette poursuite. Ces échos contribuent à tisser l'unité des Odes. Ce traitement privilégié est absent des Satires.

Ainsi, de toute évidence, la satire est plus familière, moins « poétique » que l'ode ; elle se plaît à l'inventaire discontinu des espèces animales dont l'origine, dans le livre II, tient sans doute aux parodies du verbiage philosophique de Damasippe et d'ofellus, de la logorrhée culinaire de Catius. Horace se défend d'être l'écrivain-fleuve Lucilius mais il se laisse quelquefois séduire par la virtuosité de l'énumération. L'animal satirique semble avant tout un objet au service de la diatribe morale. Les animaux des Odes, eux, ont essentiellement une fonction descriptive et poétique ; dans certaines comparaisons développées, ils atteignent presque au statut de personnage : c'est ainsi que dans l'ode I, 23 les frémissements des feuilles mouvantes aux souffles du printemps ou l'écartement des ronces au passage des lézards verts sont décrits selon la perception subjective du faon craintif.

\section{Appliquons désormais notre analyse quantitative à chaque animal}

Pour plus de commodité, je vais garder la classification de Pline l'Ancien. Les quatre grandes espèces: Les animaux terrestres sont les plus représentés: 25 animaux différents apparaissent 136 fois; puis viennent les oiseaux: 19 espèces pour 33 apparitions ; ensuite les animaux aquatiques : 15 espèces pour 30 apparitions ; enfin les insectes dont trois représentants se manifestent 3 fois. Cette hiérarchie des proportions correspond grossièrement à celle du catalogue plinien (animaux terrestres : 229§ ; animaux aquatiques : $186 \S$; oiseaux : $212 \S$; insectes : $120 \S)$. Le peu d'intérêt d'Horace pour les insectes s'explique, sans doute, d'un côté par l'absence d'anthropomorphisme qui réduit les possibilités de comparaison et de métaphores, de l'autre par le faible avantage et le moindre danger qu'ils présentent pour l'homme. Ce sont les animaux aquatiques qui se taillent la part « du lion » dans les Satires : 15 espèces, 30 apparitions, contre 3 mentions dans les Odes où l'on ne mange ni poissons, ni coquillages, ni crustacés. Car on ne mange pas dans les Odes; à la place, on sacrifie des animaux, occupation plus relevée. A l'inverse, 20 espèces terrestres apparaissent 94 fois dans les Odes contre 42 fois dans les satires, indice vraisemblable d'un réseau construit de significations. Les représentations des oiseaux et des insectes sont plus équilibrées avec, respectivement, 13 et 2 espèces dans les Satires contre 9 et 1 espèces dans les Odes, 18 et 2 apparitions dans les Satires contre 15 et 1 apparitions dans les Odes.

\section{Existe-t-il des animaux « lyriques »?}

51 Figurent uniquement dans les Odes: les cerfs (6 occurrences), les tigres (4), le cygne (3), la corneille (2), les lynx (1), les ours (1), les lézards (1), le daim (1), l'orfraie (1), le pivert (1), le ramier (1), l'épervier (1) , l'abeille (1)

52 Figurent majoritairement dans les Odes : les chevaux (15 occurrences contre 2 dans les satires), les bovins (taureaux, bœufs, vaches, génisse, veau : 13 occurrences contre 4), les lions (10 occurrences contre 1), les serpents (9 occurrences contre 1$)$, les loups (5 occurrences contre deux), les caprins (chèvres, chevreaux et boucs : 5 occurrences contre 1).

\section{Existe-t-il des animaux « satiriques »?}

54 Figurent uniquement dans les Satires: le turbot (4 occurrences), le mulet, les coquillages en général, les poules (3), les rats, les oursins, les squilles (2), les escargots, 
la grenouille, le chevreuil, le thon, l'esturgeon, le loup de mer, le scare, la plie, les murènes, les peignes, les huîtres, les paons, les oies, les grues, les cigognes, les grives, les merles, les rossignols, les plongeons, les lagopèdes, les mites et teignes (1).

Figurent majoritairement dans les Satires: les poissons en général (8 occurrences contre 2 dans les Odes), les sangliers ( 6 occurrences contre 2$)$, les lièvres (3 occurrences contre 1),

Les animaux « transgénériques »

Ce sont les bêtes fauves en général (1 occurrence dans les Satires pour 2 dans les Odes), les chiens ( 5 occurrences dans les Satires pour 6 dans les Odes), les ovins ( 7 occurrences dans les Satires pour 8 dans les Odes), les porcs ( 1 occurrence dans les Satires pour 2 dans les Odes), les renards ( 1 occurrence dans les Satires pour 2 dans les Odes), le murex (1 occurrence dans les Satires. pour 1 dans les Odes), les oiseaux en général (3 occurrences dans les Satires pour 4 dans les Odes), les pigeons (1 occurrence dans les Satires pour 2 dans les Odes).

Ainsi, par rapport à la liste du catalogue plinien, il est clair que les buts d'Horace sont fort différents : point de recherche chez lui du spectaculaire, du prodigieux, ou de l'exotisme qui, lorsqu'il est présent, se réduit aux fauves bien connus de la proche Numidie, aux poneys et aux brebis gaulois. Ce sont les Satires qui comportent le plus d'animaux spécifiques, indice d'un genre littéraire plus fermé, surtout dans le cas du livre II qui imite ouvertement la satire lucilienne du repas ridicule et du luxe, ou les pastiches de la littérature de cuisine déjà mis au goût romain par Ennius dans les Hedyphagetica. Les animaux lyriques, eux, ressemblent fort aux animaux épiques (chevaux de guerre et de chasse, fauves guerriers, animaux sauvages objets d'une chasse aristocratique et donc guerrière), aux animaux bucoliques (bovins et caprins), aux animaux érotiques (jeunes pouliches, génisses, chèvres ou autres juments en folie), indice d'un genre littéraire plus ouvert, ondoyant et divers, en train de se créer à Rome, et dont Horace se proclame l'inuentor latin. Quant aux animaux transgénériques, il sera nécessaire d'étudier si leur traitement est identique d'un genre à l'autre ou si le genre impose sa marque ; mais ceci relève de l'étude qualitative que je vais aborder à présent.

\section{3-L'analyse qualitative}

59 La richesse du sujet se découvrant au fur et à mesure de ma recherche, mais l'espace qui m'était imparti se réduisant à l'inverse, dans les mêmes proportions, j'ai choisi de ne présenter que trois points de vue afin de rendre compte, de manière sommaire, de la qualité des représentations animales chez Horace. Je me suis limitée à une approche théorique ou philosophique, puis pratique et morale, enfin poétique de la question.

\section{L'approche philosophique : quelle conception Horace se fait-il de l'animalité ?}

L'animal suit son instinct de conservation, de manière immédiate et identique dans chaque espèce ; son comportement est limité puisqu'il ne possède pas la raison libre qui choisit d'elle-même. Certes, le poète a soin de motiver les épithètes de nature; c'est ainsi que les daims craintifs, par nécessité de leur nature, le sont également par nécessité de circonstances lors des inondations cataclysmiques de l'Ode I, 2. Il n'en 
demeure pas moins que le lion est toujours un prédateur furieux (Odes I, 16 ; I, 23 ; III, 2 ; III, 11 ; III, 27), que les poissons nagent, les oiseaux volent, les daims s'enfuient en courant (Odes I, 2), le cerf également fuit toujours craintif (Odes I, 15 ; II, 16 ; III, 5 ; III, 12) de "mère en fille" (Odes I, 23) comme le lièvre ou les colombes devant les prédateurs(Odes I, 37); l'épervier attaque en vrille (Ibidem); le taureau encorne (Satires II, 1), le loup mord (Satires II, 1), le verrat frappe obliquement (Odes III, 22) et la truie est vorace(Odes III, 23) ; le corbeau se contente de guetter les proies sans défense (Satires II, 5) ; génisses, taureaux (Odes II, 5 ; II, 8), juments (Odes I, 25), chevreaux et chèvres (Odes III, 13 ; III, 15), à une époque donnée, se ruent à l'amour'. Même l'animal dressé ne peut avoir qu'une fonction unique : le cheval porte ou tire son cavalier à la guerre, à la chasse, à la course (Satires II, 1; II, 2 ; Odes I, 8 ; I, 15 ; II, 16 ; III, 7 ; III, 24) ; le chien, quand il ne s'acharne pas sur sa prise en ancien loup qu'il est (Satires II, 6), garde, y compris Cerbère (Odes II, 13 ; II, 19 ; III, 11 ; III, 16). Cet ordo naturalis inébranlable, dont le bouleversement est choisi comme une allégorie du désordre impie des guerres civiles dans l'Ode I, 2, relève sans doute d'une conception stoïcienne de l'animal. ${ }^{8}$

61 Cependant, l'animal horatien a en commun avec l'homme deux facultés psychiques irrationnelles, le thumoeides (élément courageux), celui que Prométhée a versé dans la poitrine du lion et de l'homme (Odes I, 16) et l'epithumêtikon (élément désirant). On voit ainsi les chevaux de guerre suer dans l'effort comme leurs cavaliers ou fuir sous l'emprise de la même épouvante (Odes I, 15 ; II, 1). On voit les mères redouter pour leurs jeunes taureaux non les dangers de la guerre mais les effluves sensuels de la jeune fillegénisse (Odes II, 8). C'est un point de vue que l'on peut rapprocher de celui de Platon'.

Enfin, les animaux horatiens peuvent être capables d'une certaine forme d'intelligence qui est en rapport avec la mémoire d'une situation donnée et qui instaure une différence chez des individus ou des espèces mieux doués que d'autres. Le sanglier marse de l'Ode I, 1 sait qu'il doit rompre les filets bien tressés ; si le thon de la Satire II, 5 est stupide, d'autres poissons savent ronger l'appât et échapper au piège (Ibidem). Ce serait le point de vue d'Aristote ${ }^{10}$.

Comparaisons et métaphores semblent pourtant démontrer une forte ressemblance sur le plan du comportement, entre les hommes et les animaux; ces ressemblances sont particulièrement sensibles dans les fables, celle du rat des villes et du rat des champs de la Satire II, 6 et celle de la grenouille qui veut se faire aussi grosse que le bœuf de la Satire II, 3. Les animaux y sont fortement humanisés et ne conservent que quelques aspects de leur morphologie. Toutefois, il faut tenir compte, dans ce cas précis, des effets esthétique et humoristique de décalage recherchés par un poète qui vise à plaire tout autant qu'à instruire : le rat des champs hospitalier offre du lard à demi-rongé que le rat des villes effleure d'une dent dédaigneuse ; pour la jeune grenouille le veau est un fauve énorme.

D'autre part, métaphores et comparaisons animales sont le plus souvent dégradantes pour l'homme; émotions et passions impulsives sont l'animalité de l'homme, de la femme en particulier (Satires II, 5; II, 8; Odes II, 8; III, 10; III, 20). Quand l'hypocoristique «mon petit chien" (Satires II, 3) est employé pour appeler un jeune enfant, c'est pour le faire obéir en l'amadouant puisqu'il n'est pas sensible aux arguments de la raison; quand Bacchus triomphe du géant Rhétus dans l'Ode II, 19, Horace précise qu'il l'a effrayé avec la mâchoire et les ongles effrayants du lion suggérant ainsi que le dieu n'a que certains traits physiques du lion mais non son caractère coléreux. En revanche, Octave-Auguste n'est pas à l'abri de comparaisons 
équivoques aux potentialités critiques. On le constate dans deux exemples: dans la Satire II, 1, trois détails réalistes transforment Octave en poulain non apprivoisé « mes paroles n'iront point trouver leur chemin à travers l'oreille dressée de César; si on le caresse mal à propos, il bronche car il se garde de tous côtés "; rapprochée de l'Ode III, 11 « ... dites des accents auxquels Lydé prête ses oreilles rebelles, Lydé qui, telle une pouliche de trois ans, dans les vastes plaines, bondit et s'ébat et craint d'être touchée ", cette métaphore souligne la jeunesse inexpérimentée de celui qui n'est pas encore " maître de lui comme de l'univers »; dans l'Ode I, 37 (p. 27), « comme fond l'épervier sur les timides colombes ou, sur le lièvre, le chasseur agile dans les plaines de la neigeuse Hémonie», une première comparaison homérique guerrière et tragique assimile Octave à Achille; mais elle est doublée d'une seconde comparaison callimaquéenne érotique "palimpsestueuse » qui transforme Octave en amoureux de Cléopâtre, double objet du désir de César et d'Antoine.

Pourtant, un exemple retourne cette distinction hiérarchique entre l'homme et l'animal: il y est dit qu'un homme devrait réfléchir et dominer sa passion comme le fait un animal sauvage (Satires II, 7). Cette remarque est faite par le paysan-épicurien Ofellus : mais, dirait plus précisément Epicure, les animaux n'ont pas à normer leur plaisir parce qu'ils ne connaissent aucun désir qui ne soit pas naturel et ne cherchent pas du plaisir au-delà de la satisfaction du besoin.

Ainsi, la poésie horatienne présente un point de vue philosophique éclectique, qui n'est pas lié au genre littéraire puisque les exemples donnés relèvent indifféremment des satires ou des odes. S'y manifeste une certaine familiarité de l'homme et de l'animal. Cette familiarité «zoologique » débouche-t-elle sur une éthique ? L'homme a-t-il des obligations morales envers les animaux ? La réponse à ces questions fera l'objet de mon deuxième point.

\section{L'approche pratique et morale}

67 De manière peu originale, Horace classe les animaux soit d'après les dangers que diverses espèces font courir à l'homme tels les serpents, les ours, les lions, les tigres, les loups dont la dangerosité est souvent soulignée par une épithète de nature ou d'origine, soit d'après les fonctions que les animaux assument à l'avantage de l'homme, qu'ils lui fournissent des ressources ou qu'ils lui rendent des services.

En premier lieu viennent les produits alimentaires, les viandes des gibiers, des animaux d'élevage, la chair des poissons, des crustacés et des coquillages. Horace ne s'insurge jamais contre la mise à mort de l'animal-nourriture; il n'envisage pas même qu'il puisse souffrir, telle la poule plongée vivante dans du Falerne mélangé d'eau de la Satire II, 4. C'est avec la même indifférence qu'il mentionne les animaux de sacrifice alors qu'il respecte scrupuleusement les règles du rituel: en particulier l'Ode III, 13 offre le spectacle du jeune chevreau teignant de la rougeur de son sang le cours limpide et brillant des eaux glacées de la fontaine de Bandousie ; si dans la Satire II, 3 le sacrifice d'Agamemnon à Aulis est signe de folie, c'est qu'il a confondu sa fille gnata avec une agnelle de sacrifice agna.

Cependant, Horace s'élève contre une exploitation abusive, contre-nature, de la nature elle-même pour assouvir les faux besoins d'une humanité dépravée : l'Ode I, 2 montre les poissons qui sentent les plaines liquides se rétrécir sous les blocs jetés dans la mer; les Satires II, 2 ; II, 3 ; II, 4 décrivent le gros temps assombrissant la mer pour défendre 
les poissons, alors que le pêcheur arrache inconsidérément ces derniers à une mer orageuse, que le cuisinier fait une rafle sur un étal de poissons coûteux. Les détails ostentatoires concernant les lieux ou les époques de production (Satires II, 4; Odes I, 31; II, 16), les conditions d'élevage (Satires II, 4 ; II, 8), les modes de préparation (Satires II, 8), de cuisson (Satires II, 4), les méthode de découpage (Satires II, 8), le choix arbitraire des morceaux comestibles (Satires II, 4; II, 8), par leur accumulation, soulignent l'absurdité des conduites humaines. Le raffinement de l'expression poétique, jouant sur le contraste burlesque entre le fond et la forme, dénonce également ce luxe illusoire de la table; les exemples en sont nombreux. J'en citerai un : le "grand mulet étendu dans un grand plat» de la Satire II, 2, v. 39, offre, avec ses polyptotes et son rythme spondaïque ${ }^{11}$, une parodie dégradante zoomorphique de la figure homérique du guerrier mort étendu. Ce sont essentiellement les Satires qui sont concernées par cette dénonciation du luxe inutile en matière de nourriture mais un exemple fourni par l'ode III, 16 où le poète refuse le miel produit par les abeilles de Calabre montre la permanence de la réflexion horatienne sur ce point. D'ailleurs, la dénonciation des richesses inutiles se manifeste, dans la poésie lyrique, par l'opposition entre les sacrifices modestes du sage - poète et les hécatombes ostentatoires du riche ou de l'homme politique (Odes II, 14; II, 17). Ainsi, Horace insiste sur les déviations qui apparaissent dans les rapports de l'homme au monde animal quand ils sont gouvernés par la convoitise et la démesure ; la brutalité, en revanche, ne le choque pas.

Un deuxième exemple de ressources animales, ce sont les matières qui servent à l'habillement, essentiellement la laine des brebis et la pourpre du murex. Ces productions apparaissent dans les Odes pour récuser de nouveau les richesses inutiles à la paix de l'âme : dans l'Ode II, 16, Horace refuse les laines deux fois teintes du murex africain, de même que dans la Satire II, 4 il dénonce le snobisme qui fait dédaigner le murex de Baies; dans l'ode III, 16, ce sont les épaisses toisons des brebis gauloises qui sont récusées.

71 Les services que l'homme reçoit des animaux sont avant tout matériels et pratiques. Horace en sélectionne quatre : le transport et la traction des biens et des personnes, la défense et la garde, la chasse et l'attaque, l'annonce des présages. Là encore, tous ces bienfaits généreusement dispensés par les animaux n'éveillent chez Horace aucun sentiment de reconnaissance, d'estime ou d'affection; en revanche, le moraliste, à nouveau, tire de la description de l'utilisation des animaux des constats sur l'évolution morale de l'homme : éloge de la jeunesse guerrière de Rome qui pratique la chasse ou la course à cheval (Odes I, 15 ; III, 7) ou, au contraire, blâme de son efféminement quand elle se complait dans les molles distractions grecques (Odes I, 8 ; III, 24), critique du luxe à la mode des poneys gaulois ou des juments de quadrige (Odes II, 16 ; III, 27)). Certes, ces derniers exemples sont pris exclusivement dans les Odes, mais ils expriment, à propos des animaux, le même point de vue anthropocentrique que les Satires.

Pourtant tout lecteur a goûté les charmes des descriptions bucoliques de la campagne horatienne. Si les représentations animales d'Horace sont si plaisantes, ce n'est point par ce que l'homme ou le philosophe a vécu ou pensé différemment les rapports traditionnels de hiérarchie et d'exploitation entre l'homme et l'animal, c'est parce que le poète a intégré ces représentations animales dans un monde symbolique et poétique. 


\section{L'approche poétique}

73 «Si la Muse », dit Horace dans l'Art poétique aux vers 323-324, « a accordé aux Grecs le génie, si elle leur a accordé de parler d'une bouche harmonieuse », les Latins doivent se soumettre au « lent travail de la lime ", élaguer et polir sans fin leur poème, "jusqu’à défier l'ongle le mieux coupé ». A Rome donc le texte poétique se décrit comme la copie longuement travaillée d'un objet importé et l'une des fonctions des métaphores poétiques latines, selon A. Deremetz ${ }^{12}$, c'est de dire la romanité et de la définir comme une imitation et un dépassement de la grécité.

Les animaux horatiens sont les témoins de la romanisation des sources littéraires grecques. Un des exemples les plus manifestes est, dans les Satires comme dans les Odes, la mention du lieu d'origine, romain ou italien, des animaux; se trouvent ainsi « romanisées » les imitations du traité d'Archestrate, le Dîner de Matrôn, les diatribes de Bion, les vives scènes de chasse dont le contexte érotique est imité des lyriques grecs. Les notations les plus allusives aussi bien que les vignettes descriptives les plus fraîches ou les plus développées, telles celles de la fuite amoureuse (Odes I, 23 ; II, 5) comportent le plus souvent une ou plusieurs allusions lettrées grecques que romanisent soit des réalités romaines, soit des références à des auctores latins. Ainsi, trouve-t-on dans un antipropemptikon parodique imité de la poésie érotique grecque, et servant de prétexte à un epyllion d'Europe, un catalogue humoristique d'auspicia pedestria et celestia fortement romanisés (Odes, III, 27). L'allusion lettrée euripidéenne à la chevelure serpentine des bacchantes que Bacchus attache sine fraude est romanisée par l'emploi de ce terme emprunté aux formules sacrées et juridiques (Odes II, 19) ${ }^{13}$. De même, la Satire II, 3 joue sur l'effet comique de l'insertion, dans un contexte juridique romain, de la métamorphose homérique d'un emprunteur protéiforme qui échappe aux filets de son créancier $^{14}$. Un emprunt analogue à la langue juridique romanise également les troupeaux d'Apollon dérobés par la ruse per dolum amotas dans un hymne à Mercure inspiré d'Alcée et de la tradition homérique et alexandrine (Odes I, 10) ${ }^{15}$. La forêt de Sabine et la louve monstrueuse de la guerrière Daunie transportent en Italie le topos du poète amoureux protégé de toute agression de la poésie érotique grecque ou l'allusion à l'homme bon à l'abri du danger de la poésie d'Alcée (Odes I, 22) ${ }^{16}$. La métaphore humoristique du poète transformé en chien de garde (Satires II, 1, v. 84-85)

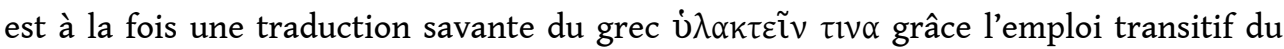
verbe latrare et une référence lettrée au Plaute romain du Poenulus (v. 1234). La longue métaphore de la jeune fille changée en génisse poursuivie par le taureau associe les souvenirs de Lucilius, Lucrèce, Catulle, Virgile, à ceux d'Anacréon et de Sappho (Odes II, $5)^{17}$. Le topos grec du sacrifice à Priape et la structure stylistique grecque neque iam, typiques du motif du retour du printemps, sont nationalisés par la présence de la divinité italique Faunus et par la mention des travaux agricoles du petit paysan (Odes I, 4).

Il existe également deux fonctions spécifiques des métaphores poétiques, toujours selon A. Deremetz : elles servent à désigner tel ou tel genre, à exprimer une préférence pour une conception esthétique particulière ainsi qu'à récuser l'excès de certaines productions poétiques; les représentations animales horatienne relèvent de ces fonctions et sont les allégories d'un art poétique. intraitables avec les serpents maures ou les lionnes gétules (Odes III, 10; III, 20) 
apparaissent dans un contexte élégiaque et leur exagération caricaturale souligne les excès de l'élégie. De la même façon, le tableau à l'esthétique pathétique de l'héroïne abandonnée au milieu des fauves, mis dans la bouche même d'Europe, propose un traitement humoristique de l'epyllion alexandrin : ne souhaite $-\mathrm{t}$ - elle pas errer nue au milieu des lions, et, belle encore, nourrir les tigres, ou, de toutes ses forces, briser les cornes du taureau qui la déshonore, ce monstre tant aimé ? Or cet epyllion parodique doit servir d'exemple à la jeune Galatée dont l'humeur est vagabonde (Odes III, 27); et pourtant, le lyrique ne la retient pas, contrairement à l'élégiaque incapable d'enfermer son désir amoureux dans une juste mesure, celle du vers comme celle de la morale épicurienne.

Conceptions morales et conceptions poétiques sont d'ailleurs associées et usent des mêmes métaphores.

Ainsi, les ailes stridentes et promptes de la Fortune rapace (Odes I, 34 ; III, 29), méprisée par le sage épicurien, sont humiliées par celles de l'âme vertueuse qui fuit les réunions du vulgaire et les marécages d'un sol bas (Odes III, 2) et par celles du poète-cygne qui emporte Horace à travers le limpide éther (Odes II, 20). Ici, certains détails descriptifs définissent précisément la poésie lyrique horatienne: inusitata, nec tenuis, biformis ; cependant les détails burlesques et zoologiques de la métamorphose du poète précisent que le genre ne doit pas être confondu avec les genres nobles traditionnels, tels l'épopée. L'image du cygne fonctionne « en réseau » et l'élément érotique des odes est signifié par l'attelage de cygnes de Vénus (Odes III, 28), tandis que leur élévation est suggérée par la comparaison implicite avec un autre poète-cygne, Varus, l'oiseau prophétique au chant méonien (Odes I, 6). Les colombes mythologiques de la campagne du Vultur apulien (Odes III, 4) qui couronnent Horace de feuillage, le consacrent poète dès l'enfance et le protègent ainsi de tous les dangers de la vie politique: elles proclament d'un côté, la suprématie des Odes comparables au lyrisme de Stésichore et de Pindare ou à la sage éloquence de Platon, et de l'autre, leur identité en tant que poésie de l'otium, c'est-à-dire célébration de la paix intérieure dont jouit, dans l'instant, le simple particulier au sein de la paix publique.

Cet idéal de l'otium, à la fois mode de vie et forme d'écriture, est exprimé dans les sacrifices d'animaux de petites dimensions, comme je l'ai signalé précédemment, et dans les tableautins idylliques de la vie campagnarde. C'est une vie plus imaginaire que réelle, où l'homme n'exploite plus ou pas les animaux (Odes III, 6 ; III, 13; III, 29), où les brebis de Galèse s'habillent de peau (Odes, II, 6), où les prédateurs fuient ou bien se promènent paisiblement, tandis que se reposent, libérées, les bêtes de somme et que joue, insouciant et sans crainte, le petit bétail dans une harmonie heureuse avec les hommes et les fauves (Odes I, 17 ; III, 18). C'est un âge d'or où se sont effacées les traces des conflits, des parjures et des convoitises des hommes, tel le site de Troie sur les tombes duquel bondissent les troupeaux tandis que les bêtes sauvages y cachent impunément leurs petits (Odes III, 3). Les Satires proposent également cet idéal de mesure sous la forme du petit champ et du petit bétail (Satires II, 2 ; II, 5), ceux du sage épicurien Ofellus ou ceux d'un Ulysse burlesque qui a perdu sa dimension épique; la Satire II, 6, v. 14-15 associe plus clairement encore l'image du petit bétail, source de richesses modestes, à une défense esthétique virgilienne et callimaquéenne du genre tenuis « Engraisse chez moi le bétail et tout le reste sauf mon esprit ». Bacchus incarnant à la fois la sagesse civilisatrice et la liberté selon un idéal stoïcien, 
soumettant les bêtes fauves (serpents, lions, tigres) sans les domestiquer (Odes III, 3) et triomphant de la mort ainsi que du chien Cerbère (Odes II, 19). Cette même puissance sur les fauves et le chien infernal est donnée à la poésie d'Alcée et de Sappho (Odes II, 13), à la lyre de Mercure protecteur d'Horace (Odes III, 11), au poète protégé des Muses (Odes III, 4) ou mieux encore, au poète libéré des dangers par le simple chant de sa poésie amoureuse (Odes I, 22). Cette ressemblance entre le pouvoir de Bacchus et celui de la poésie pourrait conforter l'existence d'un collège dionysiaque des poètes sous le patronage d'un Liber pater défenseur de l'antique liberté.

Quel bilan pouvons-nous tirer de ce survol des représentations animales dans le livre II des Satires et les livres I à III des Odes?

Les Satires, comparées aux Odes, offrent des représentations animales plus hétérogènes, parfois très fortement spécialisées et de style moins varié. Cependant, ces différences ne concernent que la forme apparente des deux œuvres. Les animaux, qu'ils soient lyriques ou satiriques, ont toujours les mêmes caractères, voire les mêmes fonctions. Apparaît également dans la poésie horatienne, sinon une défense des animaux, du moins l'apologie d'un ordre naturel contre les excès de la convoitise humaine. Dans ses descriptions suggestives des animaux en liberté de la campagne italienne s'exprime peut-être aussi la sensibilité agreste du " petit paysan de Venouse $»^{18}$. Toutefois, cette sensibilité se manifeste le plus souvent par la médiation de la doctrina littéraire, et les représentations animales du poète sont soumises à une organisation consciente des réseaux de significations dans une perspective à la fois morale et esthétique.

\section{NOTES}

Haec est finis huius enarrationis ; utinam turpiter atrum ne desinat in piscem ${ }^{19}$.

1. Les animaux d'Horace apparaissent brièvement au détour d'une œuvre plus générale. L'ouvrage de base de J.M.C. TOYNBEE, Animals in Roman Life and Art, London, 1973, cite quatorze fois Horace, mais se contente à chaque fois d'une brève allusion d'une ligne et la somme récente L'Animal dans l'Antiquité, éd. B. CASSIN, J.-L. LABARRIÈRE, Paris, 1997, ne comporte qu'une citation d'Horace. Citons pour mémoire parmi les ouvrages généraux: J. ANDRÉ, L'Alimentation et la cuisine à Rome, nouvelle édition, Paris, 1981; J. AYMARD, Essai sur les chasses romaines, Paris, 1951 ; N. BLANC, A. NERCESSIAN, La cuisine romaine antique, Grenoble, 1992 ; L. BODSON, Attitudes towards animals in Greco-Roman Antiquity, in International Journal for the Study of Animal Problems, 4, 1984, p. 3-12; Nature et statut des animaux de compagnie dans l'Antiquité gréco-romaine, in $X^{e s}$ Entretiens de Borgelat, Lyon, 1990, p. 167-174 ; Points de vue romains sur l'animal domestique et la domestication, in Homme et animal dans l'Antiquité romaine, éd. R. CHEVALLIER, Tours, 1995, p. 9-49.

Signalons enfin quelques articles spécifiques mentionnant des animaux horatiens: A. BARABINO, Immagini e metafore di animali nella scittura giambica oraziana (Hor. Epod. 5, 8 e 12), InvLuc, 15-16, 1993-1994, p. 9-29. A. BARBIERI, A proposito della satira II, 6 di Orazio, RAL, XXI, 1976, p. 479-507. L. BONFANTE, The poet and the swan: Horace Odes II ; 20, PP, XLVII, 1992, p. 25-45. I. BORZSAK, Bemerkungen zu Horazens briefen, Philologus, CXIII, 1969, p. 225-234; Non usitata, nec tenui ferar... Zu Horaz C., II, 20, ACD, XIII, 1977, p. 33-40. M.A. CERVELLERA, L'ode I, 22 di Orazio, AFLL, VII, 1975-1976, p. 41-53. G. CIPRIANI, L'ode oraziana a Cloe fra Omero e Ariosto, A \& R, XXVIII, 1983, p. 51-58. R. 
CORTÉS TOVAR, Satura, sermo y fabella en Serm. II, 6 de Horacio, in Homenaje C. Codoñer, éd. A. RAMOS GUERREIRA, Salamanca, 1991, p. 63-80. G. DAVIS, Carmina/Iambi. The literary-generic dimension of Horace's Integer vitae (C. I, 22), QUCC, 56, 1987, p. 67-78. P.J. DEHON, Horace et la chasse, Latomus, XLVII, 1988, p. 830-833. L. DESCHAMPS, Les Images du pouvoir dans les Odes d'Horace, in Images romaines du pouvoir, Eidôlon (LAPRIL), 43, 1994, p. 29-39. J.S.C EIDINOW, A note on Horace, Epistles I, 2, 26 and 2, 2, 75, CQ, XL, 1990, p. 566-568. V.A. ESTEVEZ, Chloe and the fawn. The structure of Odes I, 23, Helios VII, 1, 1979-1980, p. 35-44. E. A. FREDRICKSMEYER, Horace's Chloe (odes 1.23 : inamorata or victim ?, CJ, 89, 1993-1994, p. 251-259. D. GAGLIARDI, Ad Hor., Epist. I, 7, 29-33, Vichiana, XVI, 1987, p. 220-223. K. GANTAR, Horazens Apokynosis, Zant, XXI, 1971, p. 135-140. C. HENRIKSÉN, Vitulus iuuenescens : a note on Martial, Epigrams 9, 42, 11 and Horace, Odes IV, 2, 54-56, Eranos, 97 (1-2), 1999, p. 59-61. H. JACOBSON, Horace carm. I, 37, 26, Mnemosyne, XLIII, 1990, p. 449. D.A. KIDD, The metamorphosis of Horace, AUMLA, 35, 1971, p. 5-16. C. LECOMTE LAPP, Grues, étoiles et taureaux: images de la navigation antique, AC, 72, 2003, p. 1-23. J. McCORMICK, Horace's Integer uitae, CW, LXVII, 1973, p. 28-33. Y. NADEAU, Aenigma, an eloquens structura? Hor., C., I, 23 (uitas inuleo), Latomus, XLVI, 1987, p. 778-780. G.B. NUSSBAUM, Cras donaberis haedo (Horace Carm. III, 13), Phoenix, XXV, 1971, p. 151-159. H. N. PARKER, Fish in trees and tie-dyed sheep: a function of the surreal in Roman poetry, Arethusa, XXV, 1192, p. 293-323. C.B. PASCAL, Another look at the swan ode (carm., II, 20), Latomus, XXXIX, 1980, p. 98-108. V. PÖSCHL, Liebende Schwäne bei Horaz und später, Humanitas, 47 (1), 1995, p. 531-543. A. RICHTER, Le cygne et l'abeille. Horace, ode IV, 2, composée en 16 av. J.C., BFLM, II, 1969, p. 67-72. X. RIU, Horaci, Serm. I 10, 40-48 i la poesia bucòlica, AFB, XIV, 1991, p. 107-112. M. V. RONNICK, Green lizards in Horace: lacertae uirides in Odes 1.23, Phoenix, 47, 1993, p. 155-157. H. SCHOONHOVEN, Purple swans and purple snow (Hor., C. IV, 1, 10 and Eleg. In Maec. 62), Mnemosyne, XXI, 1978, p. 200-203. Ch. STOCCHI, Fedro, le rane e $i$ bovini: decostruzione e ricostruzione di un paradigma esopico ?Paideia, 58, 2003, p. 345-354. J. TATUM, Non usitata nec tenui ferar, AJPH, XCIV, 1973, p. 4-25. O. THÉVENAZ, Le cygne de Venouse: Horace et la métamorphose de l'Ode II, 20, Latomus, 61, (4), 2002, p. 861-888. A. G. DE TOMMASO, Media inter carmina poscunt ursum : note in margine a due frammenti di sigillata da Populania, ASNP, 25, 1995, p. 427-431. TRAINA, Il pesce epico (Hor. Sat. 2, 2, 39), MD, XXIII, 1989, p. 145-150. L.C. WATSON, The iambist as sheep-dog. Horace, Epode VI, 7-8, Mnemosyne, XXXVI, 1983, p. 156-159. J.C. YARDLEY, Horace and the wolf, Mnemosyne, XXXII, 1979, p. 333-337.

2. Sont en gras, les animaux de l'encyclopédie plinienne cités par Horace ; sont signalés par une majuscule les animaux qui font l'objet d'un ou de plusieurs chapitres de Pline, par une minuscule, les animaux qui sont cités au détour d'un chapitre; les animaux mentionnés seulement par Horace sont soulignés.

3. Le principe fondamental de Pline est de révéler la nature à travers sa variété et sa profusion ; l'accent est mis sur ce qui émerveille: mirabilia ou sur ce qui relève de la zoologie pratique, de l'utilisation des animaux par l'homme : c'est ce principe qui explique les digressions et l'ordre de présentation. Cf. L. BODSON, La conception romaine de l'animal, p. 325-331. Tous les textes latins, sauf mention contraire, sont cités d'après l'édition de la collection des Universités de France.

4. PL., H.N., VIII ; les chiffres suivants renvoient aux chapitres.

5. PL., H.N., IX ; les chiffres suivants renvoient aux chapitres.

6. PL., H.N., X ; les chiffres suivants renvoient aux chapitres.

7. Selon les épicuriens, les animaux dès leur naissance tendent spontanément au plaisir et évitent la douleur (CIC., Fin., I, 30, 71).

8. Les épicuriens, comme les stoïciens, pensent que la raison et la faculté réflexive font défaut chez les animaux. Mais ce sont les stoïciens qui ont proposé la réflexion la plus poussée sur les caractéristiques qui distinguent le comportement instinctif du comportement réfléchi et appris : ils soulignent son immédiateté (dès la naissance), sa similitude (entre animaux d'une même espèce), ses limites (chaque espèce ne connaissant qu'un ou deux moyens de conservation). Sur la conception stoïcienne de l'animal, cf. U. DIERAUER, Raison ou instinct? Le développement de la 
zoopsychologie antique, in L'animal dans l'Antiquité, p. 18-24; J.-L. LABARRIÈRE, De la "nature phantastique » des animaux chez les Stoïcines, in Passions et Perceptions. Studies in Hellenistic Philosophy of Mind, éd. J. BRUNSCHWIG, M.C. NUSSBAUM, Cambridge/MSH, 1993. Sur les différentes conceptions philosophiques antiques de l'animal, cf. CENTRO DI BIOETICA, Genova, Filosofi e animali nel mondo antico, a cura di S. CASTIGNONE e G. LANATA, Pisa, 1994 ; R. SORABJI, Animal Minds and Human Morals. The Origins of the Western debate, London, 1993.

9. Cf. J. FRÈRE, Les métaphores animales de la vaillance dans l'œuvre de Platon, in L'animal dans l'Antiquité, p. 423-434.

10. Cf. J.-L. LABARRIÈRE, De la phronesis animale, in Biologie, Logique et Métaphysique chez Aristote, éd. D. DEVEREUX , P. PELLEGRIN, Paris, 1990, p. 405-428.

11. HoR., S. II, 2, v. 39 : porrectum magno magnum spectare catino ; cf. HoM., Il., XVI, v. 776 ; XVIII, v. 26 ; Od., XXIV, v. 40.

12. A. DEREMETZ, Le Miroir des Muses. Poétiques de la réflexivité à Rome, Villeneuve d'Ascq, 1995.

13. EUR., Bacch., v. 99 et v. 695. Sur l'insertion de règles juridiques comme prétexte à un jeu littéraire parodique ou comme participation à une création littéraire originale, cf. M. DUCOS, Horace et le droit, REL, 72, 1994, p. 79-89.

14. Hoм., Od., IV, v. 417-418.

15. ALc., fr. 308, L.- P. HoM., Il., XXIV, v. 24 ; v. 333 ; v. 343 ; v. 444 ; v. 544 ; Od., XIX, v. 395 ; XXIV, v. 9. Sur le traitement alexandrin de l'ode, cf. G. PASQUALI, Orazio lyrico, Firenze, 1964 (2 $2^{\text {ème }}$ édition), p. 63 et suivantes. Sur la forme juridique de la tournure, cf. GAI., Inst., III, 195 ; ULP., Dig., $29,2,71,6 ; 47,9,3,5$.

16. AlC., fr. 130, L. - P. ; PosidiPP., A.P., V, 213 ; PHILOD., A.P., V, 25.

17. ANACR., fr., 417, P. ; SAPPH., fr., 96, L. - P. ; LUCIL., v. 1041, M. ; LUCR., IV, v. 1255 ; CATUL., 68, v. 126 ; VIRG., G., III, v. 169, 209, 244.

18. C'est ainsi que le définit L. DESCHAMPS dans l'article sur les images du pouvoir dans les Odes où elle analyse, occasionnellement, quelques représentations animales.

19. D'après HOR., P., v. 3-4.

\section{RÉSUMÉS}

Les Satires, comparées aux Odes, offrent des représentations animales plus hétérogènes, parfois très fortement spécialisées et de style moins varié : la satire se plaît à l'inventaire discontinu des espèces animales et l'animal satirique y est avant tout un objet au service de la diatribe morale, tandis que les animaux des Odes ont essentiellement une fonction descriptive et poétique et atteignent presque au statut de personnages. Cependant, ces divergences ne concernent que la forme apparente des deux œuvres et, d'ailleurs, la différence quantitative du nombre d'animaux, en valeur relative, entre les Satires et les Odes est moins grande qu'il n'y paraît en valeur absolue. Les animaux, qu'ils soient lyriques ou satiriques, ont toujours les mêmes caractères, sinon les mêmes fonctions. De manière peu originale, Horace classe les animaux soit d'après les dangers que diverses espèces font courir à l'homme, soit d'après les fonctions que les animaux assument à l'avantage de l'homme. La poésie horatienne, quel que soit le genre, présente également une conception philosophique éclectique de l'animal. Cependant, Horace s'élève contre une exploitation abusive, contre-nature, de la nature elle-même pour assouvir les faux besoins d'une humanité dépravée. Dans ses descriptions suggestives des animaux en liberté de la 
campagne italienne s'exprime peut-être aussi la sensibilité agreste du petit paysan de Venouse . Toutefois, cette sensibilité se manifeste le plus souvent par la médiation de la doctrina littéraire, et les représentations animales du poète sont soumises à une organisation consciente des réseaux de significations dans une perspective esthétique : les animaux horatiens contribuent ainsi à la romanisation des sources littéraires grecques et à la défense d'un art poétique particulier.

The Satires, compared with the Odes, present more heterogeneous animal representations, sometimes strongly specialised and in a less varied style : the satire takes pleasure in making a discontinuous assessment of animal species and the satiric animal is there, before all, an object in the service of a moral diatribe, while the animals in the Odes have essentially a descriptive and poetic function, and almost reach the status of characters. These divergences concern only the apparent form of the two works and, besides, the quantitative difference in number of animals, in relative value, between the Satires and the Odes is not as big as it appears in absolute value. The animals, wether they are lyrical or satirical have always the same nature, if not the same functions. In a not very original way, Horace classifies the animals wether by the great dangers in which the diverse species put the men, or by the functions that the animals assume to the advantage of men. The poetry of Horace, whatever its genre, presents also a philosophical eclectic conception of the animal. However, Horace rises up against an abusive and unnatural exploitation of nature itself, to satisfy the false needs of a depraved humanity. In his suggestive descriptions of the animals roaming free in the italian country, perhaps also expresses itself the rustic sensibility of a small peasant from Venusia. Nevertheless, this sensibility appears, more often, thanks to the mediation of the literary doctrina, and the animal representations of the poet are subjected to a conscious organisation of networks of significations in an aesthetic perspective. So, the animals of Horace contribute to romanization of the greek literary sources and the defence of a special poetic art.

\section{INDEX}

Mots-clés : animal, catalogue, genre, Horace, Odes, poétique, représentation, Satires

\section{AUTEUR}

\section{DOMINIQUE VOISIN}

Maître de Conférences de Latin à l'Université de Nice 